\title{
A Subcritical Reactor with Electron Linacs for Transmutation of Nuclear Wastes
}

\author{
$\dagger$ - A. Krasnykh, Yu. Popov, V. Rudenko, and L. Somov, \\ †- L. Men'shikov, V. Prusakov, and S. Subbotin \\ $\dagger$ - Joint Institute for Nuclear Research, Dubna, Russia and \\ $\ddagger$ - Russia Research Center "Kurchatov Institute", Moscow \\ email: krasnykh@main1.jinr.dubna.su
}

\section{REACTOR TRANSMUTATOR}

\section{Abstract}

Subcritical Actinide Nuclear Reactor (SCANUR) guided by the electron - gamma -neutron chains is considered. It incinerates the nuclear wastes of one nuclear power plant with power about $3 \mathrm{GW}$. The main requirements for the reactor system and electron linac are formulated. In this paper the outline of the main linac system and parameters is discussed.

\section{INTRODUCTION}

The conception of the melted salt subcritical reactor for the transmutation of high actinides such as ${ }^{241} \mathrm{Am},{ }^{243} \mathrm{Am}$, $\ldots$ and including longlived ${ }^{129} \mathrm{I}$ is considered. The fuel of SCANUR is melted salt of fluoride actinides that circulate in the external loop by thermal convection. The actinide fission fragments are extracted from the loop by the radiochemical method. Simultaneously the heat removal by the external loop is allowed. The total average e-beam power needs about 1.5-4 MW for transmutation of nuclear wastes from one block of a plant with power $3 \mathrm{GW}$. The reactor version with the effective neutron multiplication coefficient $k_{e f f}=0.997$ is discussed. The experimental results and the experience gained at the existing melted salt reactors are taken into account here. The SCANUR is driven and fed by the external neutron source which it is based on the system of S-band linacs. The linacs are situated in the horizontal plane around the active section (core). This design solves the problem of injecting the e-beam into reactor. The well known pulse rf-technology is used in the SCANUR conception. Most of the rf-equipment which is required to build the linac system is a part of well developed technology or could be scaled from systems that have been running for many years.

-This work was cared out as a hobby and by authors' enthusiasm without any support.
Our point of view is the following. All actinide fission fragments besides ${ }^{129}$ I should be buried. Only high actinides ${ }^{241} \mathrm{Am},{ }^{243} \mathrm{Am},{ }^{237} \mathrm{~Np}, \ldots$ and ${ }^{129} \mathrm{I}$ should be incinerated in SCANUR. Plutonium should be return back to the nuclear plant. So, SCANUR must incinerate $J_{a}=3 \cdot 10^{18}$ nuclei per second for the $3 \mathrm{GW}$ power plant. The total cross section for $n, \gamma$ and $n, f$ reactions with actinides is rather high (about 500 barn). The wastes could be incinerate in a conventional slow reactor but this way is connected with the high nuclear hazard. The subcritical melted actinide fuoride salt reactor for transmutation of nuclear wastes has a whole row of the advantages. The main one is the guaranteed safety when there is a high level of the effective neutron multiplication coefficient. The reactor fuel is a liquid melted salt of the fluoride actinides. There are of this kind experimental running reactors but with other salt as a fuel. The designed reactor has a closed external contour. The melted salts circulate by thermal convection. The heat and product of nuclear reaction are removed from the active zone by the natural convection process. The soft driving and tuning of SCANUR is achieved by the changing of the salt concentration. There is a dilution process in the ${ }^{6} \mathrm{LiF}$ salt. As a result, there is radiation safety of the SCANUR operating with a high level of the reliability and with the effective neutron multiplication coefficient up to

$$
k_{\text {eff }}=1-\beta=0.997
$$

where $\beta$ is a fraction of slow neutrons for actinides.

It should be mentioned that

$$
k_{e f f}=1-10 \cdot \beta
$$

for the solid fuel reactors. The SCANUR has a high level of neutron multiplication (about 300 ). In this case a simple, cheap and existing electron linac system could be adopted to solve the nuclear transmutation problem. As it will be shown bellow $k_{e f f}$ will determine the general parameters for the SCANUR linac. 


\section{Conception of Electron LINAC FOR TRANSMUTATION OF WASTES}

The needed average e-beam powet $P_{a v}$ is:

$$
P_{a v}=J_{a}\left(1-k_{e} \int\right) \nu C_{n}
$$

where:

$\nu \simeq 3$ is the neutron multiplicity for actinides;

$C_{n}=E_{e} / \chi_{n}$ is the "neutron price";

$E_{e}$ is the incident energy of the e-beam and

$\chi_{n}$ is efficiency of the photonuclear reaction for neutrons that were born inside the active zone.

The neutron price for the $\sim 100 \mathrm{MeV}$ incident e-beam is $\simeq 1.1 \mathrm{GeV}$ for natural uranium. The price for high actinides will be $\simeq 0.8 \mathrm{GeV}$. So, the total average beam power should be up to $\simeq 4 \mathrm{MW}$ for the $3 \mathrm{GW}$ nuclear plant and SCANUR would incinerate about $J_{a}=3 \mathrm{i} 0^{18}$ nuclei per second. It is clear from (1) that the total beam power is determined by the effective neutron multiplication coefficient $k_{e j f}$. Different kind of accelerators (linear and cyclic, conventional linacs and new ones, induction or rf-linacs, superconducting or warm rf-linacs etc.) were discussed and the analysis was carried out. Their comparison with a proton linac was carried out of course. The analysis showed us that the S-band e-beam linac could have a number of advantages (for this time) as compared with others. The main advantage of this combination of an e-beam linac and a liquid melted salt of the fluoride actinides reactor is a total low cost (the final price that include $R \& D$ and operation cost, safety, time of running, ecology etc.) and its "chamber" and proper relation to the nuclear block of plant.

The needed total beam power is too high to be obtained by only one electron linac but it could be spread in space and time. The preliminary estimates was showed, for example, that the perimeter of the SCANUR active zone in the horizontal plane could be about $6 \mathrm{~m}$. For the SCANUR project could be used a several separate S-band linacs. The total number of linacs is 16 , for example. Each one could have conventional (not record!) parameters. The linac system is based on the use of well developed technology that have been in successful operation for a many years. It seems to us that a SLAC-like $2 \pi / 3$ traveling wave constant gradient structure can be used. It is more cheaper relative $\mathrm{L}$-band stuctures. Instead of the single bunch operation mode, the SCANUR linac should get the multibunch one.

SCANUR, for example, has got of 16 linacs, which are situated around the active zone. The output target is made of tantalum, which is more suitable for a high level of average $e-, \gamma, e+$ and $n$ power fluxes. The general parameters of the SCANUR linac system are presented in Table 1.

The operation cyclogram was chosen as follows in order to decline the target heating problems. Linac operates consistently and in turn. The pulse length of each electron beam is about $4.15 \mu \mathrm{sec}$. The total illumination time of

\begin{tabular}{|c|c|}
\hline Parameter & Value \\
\hline Energy & $112 \mathrm{MeV}$ \\
Average beam power & $1972 \mathrm{~kW}$ \\
Linac length (each one) & $\sim 25 \mathrm{~m}$ \\
Number of linacs & 16 \\
Repetition rate & $600 \mathrm{~Hz}$ \\
Linac efficiency & 0.74 \\
\hline
\end{tabular}

Table 1: SCANUR Linac Parameters

\begin{tabular}{|c|c|}
\hline Parameter & Value \\
\hline RF-frequency & $2856 \mathrm{MHz}$ \\
Pulse duration of rf-power & $\simeq 5 \mu \mathrm{sec}$ \\
Beam pulse length & $\sim 4.15 \mu \mathrm{sec}$ \\
Average output power & $123 \mathrm{~kW}$ \\
Average beam current & $0.0011 \mathrm{~A}$ \\
Average pulse current & $0.46 \mathrm{~A}$ \\
Pulse beam current & $5.4 \mathrm{~A}$ \\
Energy of beam & $112 \mathrm{MeV}$ \\
Bunch charge & $161 \mathrm{pC}$ \\
Bunch length & $3 \mathrm{~mm}$ \\
Distance between bunches & $10.5 \mathrm{~cm}$ \\
\hline
\end{tabular}

Table 2: The Main SCANUR Linac Parameters

the target is about of $70.5 \mu \mathrm{sec}$ followed by a relatively long (about $1.6 \mathrm{msec}$ ) pause for the technological process in the SCANUR active zone. The EGS3-code was used to estimate the energy deposition on the target. It should be mentioned that there is a possibility of using the coherent bremsstralung from the crystal target for to get of the additional gain for the neutron flux. The using of crystal target allow to reduce some the neutron price for SCANUR. The estimates of coherent bremsstralung is shown that for $E_{e} \simeq 40 \mathrm{MeV}$ the neutron price could reach up to $C_{n} \simeq 300 \mathrm{MeV}$. Than the total average power could decline to $1.5 \mathrm{MW}$.

It seems to us that accumulation of the defects in the crystal converter target is not so much significant because there is a continuous annealing process by electron beam. The value of the gain should be specified by experiment in the nearest future. The using of the coherent bremsstralung from the crystal target could be useful and for design of positron source for electron/positron colliders with a high average luminosity. This is the important moment both for the SCANUR project and the design of positron sources with a high yield.

In Table 2 was given the main parameters for one linac.

There was the analysis of several variants of the SLAClike structures in the range $a / b=0.25 \div 0.35$ where $a$ is the beam hole radius and $b$ is the inner radius of the accelerating structure. The aim of the study was to determinate the beam loss parameter for the operation and HOM frequencies. The broad band of the structure is an important parameter for the multibunch operation mode. It was studied by the URMELT and TBCI codes. It was interested to compare the URMELT dates with dates that had get from Handbook on Disk Loaded Structure by O.Val'dner 


\begin{tabular}{|c|c|c|c|}
\hline $\mathrm{a} / \mathrm{b}$ & 0.24 & 0.3 & 0.35 \\
Transmittance for $f_{0}$ & $12 \mathrm{MHz}$ & & $56 \mathrm{MHz}$ \\
Transmittance on $f_{H O M}$ & $49 \mathrm{MHz}$ & $105 \mathrm{MHz}$ & $198 \mathrm{MHz}$ \\
$k_{\text {loss }}$ for $f_{0}$ & $1.1 \mathrm{~V} / \mathrm{pC}$ & $0.88 \mathrm{~V} / \mathrm{pC}$ & \\
$k_{\text {loss }}$ for $f_{\text {HOM }}$ & $0.088 \mathrm{~V} / \mathrm{pC}$ & $0.1 \mathrm{~V} / \mathrm{pC}$ & $0.3 \mathrm{~V} / \mathrm{pC}$ \\
\hline
\end{tabular}

Table 3: Comparison of Linac Structures

and et al. In Table 3 some data are presented.

The structure with $\mathrm{a} / \mathrm{b}=0.3$ could get the next stage of the SCANUR linac design. There is not so bad agreement between computer and the Handbook data for the operating frequency. The different is not more than $8 \%$. The different increase when the dipole mode parameters are calculated The thermoproblem of the disk will be considered on the next stage of the study.

The Russian klystron "Bereg" could be used as the pulse rf-power supply. It is a multibeam high pulse power klystron. The output pulse power is of $10 \mathrm{MW}$ and the average power is about $25 \mathrm{~kW}$. The pulse length of $\mathrm{rf}$-power is $5 \mu \mathrm{sec}$. The anode voltage is rather low (about $80 \mathrm{kV}$ ) and the pulse power supply is not so large as for the single beam tube power supply. This fact could be very useful for the SCANUR linac.

The beam loading problem for the multibunch operation mode was also studied for optimization of the energy spectrum. The energy spectrum of the beam would be to have the minimum if the injection start had shifted before the time of the rf-filling $0.335 \cdot t_{f}$ were $t_{f}$ is the filling time of the section (it is about $0.83 \mu \mathrm{sec}$ for the standard SLACsection). In this case the energy spectrum will be no more than $14 \%$. The preliminary study of the transverse wake fields (TBCI-code) for the SCANUR linac regime gives the hope that there will not be any problems for the beam stability with the gradient about $5.3 \mathrm{MeV} / \mathrm{m}$ and accelerating train of bunches with $\sigma_{z}=3 \mathrm{~mm}$ and $160 \mathrm{pC}$ per bunch.

\section{Conclusion}

The use of the combination subcritical melted actinide fluoride salts reactor and S-band linac system for the transmutation of nuclear wastes would allow to large reduce the cost of $\mathrm{R} \& \mathrm{D}$ for the solving problems of the incineration of the longlieved nuclear wastes of the plants. The SCANUR systems is rather compactly relative the system of the nuclear plant and could place on the direct on the plant. The most of the details and the ground system that are required to start the experimental study of the transmutation nuclear wastes are a part of well development technology. In this case there is a possibility to start the draft proposal for the solving this problem. 